\title{
Front Matter: Volume 10874
}

, "Front Matter: Volume 10874," Proc. SPIE 10874, Optical Tomography and Spectroscopy of Tissue XIII, 1087401 (12 April 2019); doi: 10.1117/12.2531772

SPIE. Event: SPIE BiOS, 2019, San Francisco, California, United States 


\title{
PROGRESS IN BIOMEDICAL OPTICS AND IMAGING
}

\section{Optical Tomography and Spectroscopy of Tissue XIII}

\author{
Sergio Fantini \\ Paola Taroni \\ Editors
}

4-6 February 2019

San Francisco, California, United States

Sponsored and Published by

SPIE 
The papers in this volume were part of the technical conference cited on the cover and title page. Papers were selected and subject to review by the editors and conference program committee. Some conference presentations may not be available for publication. Additional papers and presentation recordings may be available online in the SPIE Digital Library at SPIEDigitalLibrary.org.

The papers reflect the work and thoughts of the authors and are published herein as submitted. The publisher is not responsible for the validity of the information or for any outcomes resulting from reliance thereon.

Please use the following format to cite material from these proceedings:

Author(s), "Title of Paper," in Optical Tomography and Spectroscopy of Tissue XIII, edited by Sergio Fantini, Paola Taroni, Proceedings of SPIE Vol. 10874 (SPIE, Bellingham, WA, 2019) Seven-digit Article CID Number.

ISSN: 1605-7422

ISSN: 2410-9045 (electronic)

ISBN: 9781510623903

ISBN: 9781510623910 (electronic)

Published by

SPIE

P.O. Box 10, Bellingham, Washington 98227-0010 USA

Telephone +1 3606763290 (Pacific Time) · Fax +1 3606471445

SPIE.org

Copyright (C) 2019, Society of Photo-Optical Instrumentation Engineers.

Copying of material in this book for internal or personal use, or for the internal or personal use of specific clients, beyond the fair use provisions granted by the U.S. Copyright Law is authorized by SPIE subject to payment of copying fees. The Transactional Reporting Service base fee for this volume is $\$ 18.00$ per article (or portion thereof), which should be paid directly to the Copyright Clearance Center (CCC), 222 Rosewood Drive, Danvers, MA 01923. Payment may also be made electronically through CCC Online at copyright.com. Other copying for republication, resale, advertising or promotion, or any form of systematic or multiple reproduction of any material in this book is prohibited except with permission in writing from the publisher. The CCC fee code is 1605$7422 / 19 / \$ 18.00$.

Printed in the United States of America by Curran Associates, Inc., under license from SPIE.

Publication of record for individual papers is online in the SPIE Digital Library.

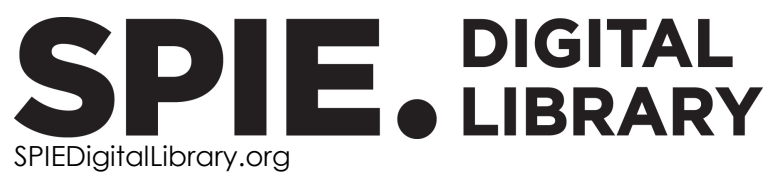

Paper Numbering: Proceedings of SPIE follow an e-First publication model. A unique citation identifier (CID) number is assigned to each article at the time of publication. Utilization of CIDs allows articles to be fully citable as soon as they are published online, and connects the same identifier to all online and print versions of the publication. SPIE uses a seven-digit CID article numbering system structured as follows:

- The first five digits correspond to the SPIE volume number.

- The last two digits indicate publication order within the volume using a Base 36 numbering system employing both numerals and letters. These two-number sets start with 00, 01, 02, 03, 04, 05, 06, 07, 08, 09, OA, OB ... 0Z, followed by 10-1Z, 20-2Z, etc. The CID Number appears on each page of the manuscript. 


\title{
Contents
}

\author{
vii Authors \\ ix Conference Committee
}

\section{ADVANCES IN METHODS AND INSTRUMENTATION I}

1087403 Self-calibrated frequency domain diffuse optical spectroscopy with a phased source array [10874-2]

1087404 RTBioT: a real-time healthcare monitoring Bio-loT device employing spatially resolved near infrared (NIR) spectroscopy (Invited Paper) [10874-3]

1087405 Toward handheld real time frequency domain diffuse optical spectroscopy [10874-5]

\section{ADVANCES IN METHODS AND INSTRUMENTATION II}

1087408 Multi-wavelength dual-detection channel system for time-resolved near-infrared spectroscopy [10874-8]

1087409 Bioresorbable fibers for time-domain diffuse optical measurements: a step toward next generation optical implantable devices [10874-9]

10874 OA Water and lipid contents measured at various parts of the human body with a six-wavelength time-resolved spectroscopy system [10874-10]

\section{DIFFUSE OPTICAL TOMOGRAPHY}

10874 OE Using dynamic vascular optical spectroscopy to evaluate peripheral arterial disease (PAD) in patients who undergo a vascular intervention [10874-14]

10874 OF New method to diagnose joints affected by systemic lupus erythematosus based on frequency-domain optical transmission [10874-15]

APPLICATIONS TO LIVER, KIDNEY, BREAST, INTESTINE

$10874 \mathrm{OH} \quad$ Near infrared spectroscopy system for quantitative monitoring of renal hemodynamics and oxygenation in rats [10874-17]

10874 Ol High spatial frequency structured light imaging texture analysis using Gabor filtering differentiates tumor from normal tissue subtypes [10874-18] 
10874 OJ Hyperspectral, hybrid continuous wave and frequency domain diffuse optical tomography in a handheld reflectance geometry for breast cancer diagnostics [10874-19]

\section{SPATIAL FREQUENCY DOMAIN IMAGING I}

1087400 Short-wave infrared spatial frequency domain imaging for non-invasive quantification of tissue water content [10874-24]

\section{SPATIAL FREQUENCY DOMAIN IMAGING II}

10874 OT A multi spectral hand-held spatial frequency domain imaging system for imaging human colorectal cancer [10874-29]

10874 OU Hand-held multi-wavelength spatial frequency domain imaging for breast cancer imaging [10874-30]

\section{FLUORESCENCE IMAGING}

10874 OV New image reconstruction algorithm for fluorescence optical tomography based on the adjoint radiative transfer equation [10874-31]

10874 OY CCD-based temperature modulated fluorescence tomography [10874-34]

\section{FLUORESCENCE AND CHERENKOV RADIATION}

$1087410 \quad$ Early detection of breast cancer using ER specific novel NIR fluorescent dye conjugate: a phantom study using FD-f-DOT system [10874-36]

$1087413 \quad$ Noninvasive imaging of dual-agent uptake in glioma and normal tissue using MRI-coupled fluorescence tomography [10874-39]

1087414 Correcting Cherenkov images for large-scale tissue-optical property attenuation using SFDI and patterned light reflectance for quantitative dosimetry [10874-40]

\section{DIFFUSE CORRELATION SPECTROSCOPY}

1087415 Diffuse correlation tomography in the transport regime: a theoretical study of the sensitivity to Brownian motion (Invited Paper) [10874-41] 


\section{CEREBRAL BLOOD FLOW}

10874 1D Dynamic measurements of absolute cerebral blood flow with coherent hemodynamics spectroscopy [10874-49]

$10874 \mathrm{IE} \quad$ Frequency dependent hemodynamic response to intracranial pressure changes [10874-50]

FUNCTIONAL BRAIN IMAGING

$10874 \mathrm{1H} \quad$ An fNIRS probe positioning system using augmented reality technology [10874-53]

$1087411 \quad$ Comparison of spontaneous and induced coherent hemodynamics in the human brain [10874-54]

\section{BREAST IMAGING}

$10874 \mathrm{lL} \quad$ Monitoring total hemoglobin concentration changes across neoadjuvant chemotherapy in PgR-positive and PgR-negative breast cancer with diffuse optical tomography [10874-57]

$108741 \mathrm{~N}$ Multi-wavelength time domain diffuse optical tomography for breast cancer: initial results on silicone phantoms [10874-59]

\section{POSTER SESSION}

10874 is Quantifying joint blood flow in a rat model of rheumatoid arthritis with dynamic contrastenhanced near-infrared spectroscopy [10874-63]

$108741 \mathrm{~A}$ A three-wavelength 240-channel NIRS-DOT system of lock-in photon-counting mode for brain functional investigation [10874-65]

10874 IV A multi-wavelength single-pixel SFD imaging system based on lock-in photon-counting detection [10874-66]

10874 IW Phantom verification for lock-in-photon-counting-based diffuse optical tomography system [10874-67]

10874 1X Anisotropy factor reconstruction as a new endogenous contrast for cancer diagnosis with optical tomography [10874-68]

$1087421 \quad$ A dual-wavelength spread spectrum-based spectroscopic system for time-domain near-infrared diffuse optical imaging [10874-72]

1087425 Spatial-temporal constraints guided dynamic fluorescence tomographic model for enhanced imaging of organs and functional structures in small animals [10874-76] 
1087427 In silico investigation of near-infrared light propagation in the joints of the human hand [10874-78]

$1087429 \quad$ Adaptive extraction of permissible source region based on matched filtering for bioluminescence tomography [10874-80]

10874 2A Depth dependent coherent hemodynamics during induced blood pressure oscillations [10874-81]

$108742 B \quad$ A miniature frequency domain diffuse optical optode for quantitative wearable oximetry [10874-82]

10874 2C An integration model of steady-state single-fiber diffuse reflectance [10874-83]

10874 2J A point-of-care handheld region-of-interest (ROI) 3D functional diffuse optical tomography (fDOT) system [10874-90] 


\section{Authors}

Numbers in the index correspond to the last two digits of the seven-digit citation identifier (CID) article numbering system used in Proceedings of SPIE. The first five digits reflect the volume number. Base 36 numbering is employed for the last two digits and indicates the order of articles within the volume. Numbers start with 00, 01, 02, 03, 04, 05, 06, 07, 08, 09, OA, OB...0Z, followed by 10-1Z, 20-2Z, etc.

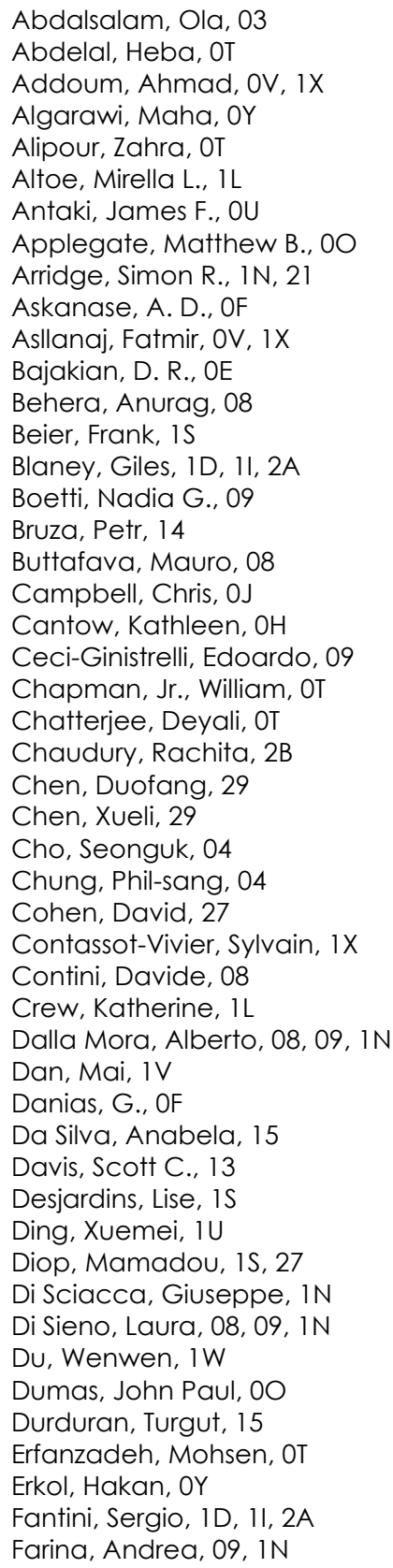


Lee, Ting-Yim, $1 S$

$\mathrm{Li}$, Jiao, $1 \mathrm{~W}$

Li, Shuying, OT

Li, Tongxin, $1 \mathrm{~V}$

Liu, Dongyuan, $1 \mathrm{U}$

Liu, Han, IW

Macdonald, Callum M., 15

Maloney, Benjamin W., Ol

Mankodiya, Kunal, 2J

Markel, Vadim A., 15

Marone, Alessandro, OE, OF, 1L, 25

Martelli, Fabrizio, $1 \mathrm{~N}$

McClatchy, David M., Ol

Mehrabi, Mehrnaz, OY

Meng, Boyu, 13

Milanese, Daniel, 09

Mimura, Tetsuya, OA

Morrison, Laura B., is

Mutch, Matthew, OT

Nandy, Sreyankar, OT

Nelson, Samantha, $1 \mathrm{E}$

Neville, K. E., OF

Niendorf, Thoralf, $\mathrm{OH}$

Nouizi, Farouk, OY

Ohmae, Etsuko, OA

O'Sullivan, Thomas D., 03, 05, 0J

Pan, Tiantian, $1 \mathrm{U}$

Papadimitriou, Konstantinos I., 21

Paulsen, Keith D., OI

Pham, Thao, 1D, 11, 2A

Piao, Daqing, 2C

Pierce, Mark C., $0 \mathrm{O}$

Pifferi, Antonio, 09, 1N

Pillai, Vinay Jha, 10

Pogue, Brian W., Ol, 14

Pohlmann, Andreas, $\mathrm{OH}$

Powell, Samuel, 21

Pugliese, Diego, 09

Ramachandran, Hema, 10

Renna, Marco, 08

Rizzo, Elizabeth J., 0 I

Robbins, Constance M., OU

Roblyer, Darren M., 0O, 2B

Ruesch, Alexander, $1 \mathrm{E}$

Sadeghipour, Negar, 13

Sahyoun, Christine C., $0 \mathrm{O}$

Saikia, Manob Jyoti, 2J

Samkoe, Kimberley S., 13

Sampathkumaran, Uma, OY

Sassaroli, Angelo, 1D, 1I, 2A

Schmitt, Samantha, 1E

Seeliger, Erdmann, $\mathrm{OH}$

Smith, Matthew A., IE

St. Lawrence, Keith, 1S

Stillwell, Roy A., 05

Strawbridge, Rendall R., 13

Streeter, Samuel S., ol

Sun, Tengfei, 2C

Suzuki, Hiroaki, OA

Suzuki, Norihiro, OA
Taroni, Paola, $1 \mathrm{~N}$

Tejada, Mariella, $1 \mathrm{~L}$

Tichaver, Kenneth, 13

Tosi, Alberto, 08

Tricoli, Ugo, 15

Tromberg, Bruce J., 04

Ueda, Yukio, OA

Vasudevan, Sandhya, OJ

Wada, Hiroko, OA

Wang, Bingyuan, $1 \mathrm{U}$

Wells, Wendy A., Ol

Xie, Jinbin, $1 \mathrm{~W}$

Yamada, Toru, $1 \mathrm{H}$

Yamashita, Yutaka, OA

Yang, Jason, OU, $1 \mathrm{E}$

Yin, Guoyan, IW

Yoshimoto, Kenji, OA

Zanoletti, Marta, 08

Zhang, Limin, IW

Zhang, Yao, $1 \mathrm{U}$

Zhang, Yide, 03

Zhao, Kuanxin, IV

Zhao, Yanyu, 00

Zheng, Yifeng, OT

Zhou, Zhongxing, $1 \mathrm{~W}$

Zhu, Quing, OT

Zhu, Shouping, 29 


\section{Conference Committee}

Symposium Chairs

James G. Fujimoto, Massachusetts Institute of Technology (United States)

R. Rox Anderson, Wellman Center for Photomedicine, Massachusetts General Hospital United States) and Harvard Medical School (United States)

Symposium Co-chairs

Jennifer K. Barton, The University of Arizona (United States)

Wolfgang Drexler, Medical University of Vienna (Austria)

Program Track Chairs

Tuan Vo-Dinh, Fitzpatrick Institute for Photonics, Duke University (United States)

Anita Mahadevan-Jansen, Vanderbilt University (United States)

Conference Chairs

Sergio Fantini, Tufts University (United States)

Paola Taroni, Politecnico di Milano (Italy)

Conference Program Committee

Robert R. Alfano, The City College of New York (United States)

Erin M. Buckley, Georgia Institute of Technology (United States)

Regine Choe, University of Rochester (United States)

Hamid Dehghani, The University of Birmingham (United Kingdom)

Amir H. Gandjbakhche, National Institutes of Health (United States)

Sylvain Gioux, Laboratoire des sciences de l'Ingénieur, de l'Informatique et de l'Imagerie (France)

Andreas H. Hielscher, Columbia University (United States)

Shudong Jiang, Thayer School of Engineering at Dartmouth (United States)

Jana M. Kainerstorfer, Carnegie Mellon University (United States)

Anand T. N. Kumar, Athinoula A. Martinos Center for Biomedical Imaging (United States)

Frederic Leblond, Ecole Polytechnique de Montréal (Canada)

Mark J. Niedre, Northeastern University (United States)

Eiji Okada, Keio University (Japan)

Thomas D. O'Sullivan, University of Notre Dame (United States)

Antonio Pifferi, Politecnico di Milano (Italy)

Anne Planat-Chrétien, CEA-LETI (France) 
Valentina Quaresima, Università degli Studi dell'Aquila (Italy)

Darren M. Roblyer, Boston University (United States)

Ilias Tachtsidis, University College London (United Kingdom)

Heidrun Wabnitz, Physikalisch-Technische Bundesanstalt (Germany)

Quing Zhu, Washington University in St. Louis (United States)

\section{Session Chairs}

1 Advances in Methods and Instrumentation I

Jana M. Kainerstorfer, Carnegie Mellon University (United States)

2 Advances in Methods and Instrumentation II

Qianqian Fang, Northeastern University (United States)

3 Diffuse Optical Tomography

Sergio Fantini, Tufts University (United States)

4 Applications to Liver, Kidney, Breast, Intestine

Mamadou Diop, Lawson Health Research Institute (Canada)

5 Spatial Frequency Domain Imaging I

Sergio Fantini, Tufts University (United States)

Paola Taroni, Politecnico di Milano (Italy)

6 Spatial Frequency Domain Imaging II

Darren M. Roblyer, Boston University (United States)

$7 \quad$ Fluorescence Imaging

Mark J. Niedre, Northeastern University (United States)

8 Fluorescence and Cherenkov Radiation

Anand T. N. Kumar, Athinoula A. Martinos Center for Biomedical Imaging (United States)

9 Diffuse Correlation Spectroscopy

Erin M. Buckley, Georgia Institute of Technology (United States)

10 Cerebral Blood Flow

Adam T. Eggebrecht, Washington University School of Medicine in St. Louis (United States)

11 Functional Brain Imaging

Stefan A. Carp, Athinoula A. Martinos Center for Biomedical Imaging (United States)

12 Breast Imaging

Paola Taroni, Politecnico di Milano (Italy) 